\title{
Benchmark calculations for ${ }^{3} \mathrm{H},{ }^{4} \mathrm{He},{ }^{16} \mathrm{O}$ and ${ }^{40} \mathrm{Ca}$ with ab-initio coupled-cluster theory
}

\author{
G. Hagen,${ }^{1,2,3}$ D.J. Dean, ${ }^{1}$ M. Hjorth-Jensen,${ }^{4}$ T. Papenbrock,,${ }^{1,2}$ and A. Schwenk ${ }^{5}$ \\ ${ }^{1}$ Physics Division, Oak Ridge National Laboratory, Oak Ridge, TN 37831, USA \\ ${ }^{2}$ Department of Physics and Astronomy, University of Tennessee, Knoxville, TN 37996, USA \\ ${ }^{3}$ Center of Mathematics for Applications, University of Oslo, N-0316 Oslo, Norway \\ ${ }^{4}$ Department of Physics and Center of Mathematics for Applications, University of Oslo, N-0316 Oslo, Norway \\ ${ }^{5}$ TRIUMF, 4004 Wesbrook Mall, Vancouver, BC, Canada, V6T $2 A 3$
}

\begin{abstract}
We present ab-initio calculations for ${ }^{3} \mathrm{H},{ }^{4} \mathrm{He},{ }^{16} \mathrm{O}$, and ${ }^{40} \mathrm{Ca}$ based on two-nucleon low-momentum interactions $V_{\text {low } k}$ within coupled-cluster theory. For ${ }^{3} \mathrm{H}$ and ${ }^{4} \mathrm{He}$, our results are within $70 \mathrm{keV}$ and $10 \mathrm{keV}$ of the corresponding Faddeev and Faddeev-Yakubovsky energies. We study in detail the convergence with respect to the size of the model space and the single-particle basis. For the heavier nuclei, we report practically converged binding energies and compare with other approaches.
\end{abstract}

PACS numbers: 21.10.Dr, 21.60.-n, 31.15.Dv, 21.30.-x

\section{INTRODUCTION}

Ab-initio few- and many-body methods have been used with great success to explore the structure of light nuclei based on microscopic two- and three-nucleon interactions. For nuclei with $A \lesssim 12$ nucleons, several techniques provide practically exact solutions to the nuclear many-body problem and have been benchmarked to agree within numerical uncertainties for the ${ }^{4} \mathrm{He}$ groundstate energy and radius obtained from the nucleonnucleon (NN) Argonne $v_{8}$ potential [1]. These methods include Faddeev-Yakubovsky equations [2], variational approaches [3, 4, 5, 6], the Green's function Monte Carlo (GFMC) method [7], the No-Core Shell Model (NCSM) [8, 9], and the effective interaction hyperspherical harmonics method [10, 11]. Many of the above approaches are, however, restricted to the lightest nuclei.

In recent years, the coupled-cluster method [12, 13] was reintroduced in nuclear physics as a tool for ab-initio nuclear structure calculations [14, 15, 16, 17, 18, 19, 20, 21]. Coupled-cluster theory is size-extensive and scales rather gently with an increasing number of nucleons and with the size of the model space, and therefore has the potential to extend the reach to medium-mass nuclei. In this paper, we address the question of whether the coupledcluster method is as precise for few-body systems as the well-established methods. Several findings suggest that this is indeed the case. Mihaila and Heisenberg performed a microscopic calculation of the electron scattering structure function for ${ }^{16} \mathrm{O}$ and found excellent agreement with experimental data. Their calculations are based on a particle-hole energy expansion of the cluster operator. More recent applications [16, 17, 18, 19, 20, 21] follow the "standard" approach of coupled-cluster calculations from quantum chemistry $22,24,25,26]$. In these calculations, several results for helium isotopes [17, 20] were found to be in good agreement with exact diagonalizations in sufficiently small model spaces and with corresponding renormalized interactions. However, except in the recent study of three-nucleon forces (3NF) in coupled-cluster theory [21], this approach has never been compared in detail to well-established few-body methods in the larger model spaces that are needed for convergence with modern NN interactions. It is the purpose of this work to fill this gap in nuclear physics, and to place the coupled-cluster method in the group of $a b$-initio approaches.

This paper is organized as follows. In Section II we begin with a brief discussion of the coupled-cluster method, of the low-momentum interactions and the employed basis spaces. Our main results for ${ }^{3} \mathrm{H}$ and ${ }^{4} \mathrm{He}$ are presented in Section III, and for ${ }^{16} \mathrm{O}$ and ${ }^{40} \mathrm{Ca}$ in Section IV. We conclude with a summary in Section $\mathrm{V}$.

\section{METHOD, INTERACTIONS, AND MODEL SPACES}

\section{A. Coupled-cluster method}

Coupled-cluster theory was invented by Coester and Kümmel almost fifty years ago [12, 13]. During the 1970s, this approach was further developed and found many applications in nuclear physics. The review by the Bochum group 27] summarizes the status of the field in 1978. From there on, applications in nuclear physics were more of a sporadic nature [28]. This was most probably due to the difficulty of hard NN interactions and their strong short-range repulsion and short-range tensor force. Mihaila and Heisenberg employed coupled-cluster theory in the late 1990s 14. Their work culminated in the precise computation of the electron scattering form factor for ${ }^{16} \mathrm{O}$ based on the Argonne $v_{18}$ potential combined with leading contributions from $3 \mathrm{NF}$ [15].

Parallel to the field of nuclear physics, coupled-cluster theory saw its own career in ab-initio quantum chemistry. After the pioneering works by Č́žek [29, 30], the theory has become one of the main workhorses in quantum chemistry 22, 24, 25, 26]. The sheer number of applications and developments in that field de-facto established a "standard" or "canonical" way for how the method is being used to solve quantum many-body problems. Reference [22] gives a summary of state-of-the-art 
coupled-cluster calculations in quantum chemistry.

Recently, coupled-cluster theory has seen a renaissance in nuclear physics starting with the calculations of Ref. [16]. This approach differs from the one by Mihaila and Heisenberg as it employs coupled-cluster theory in the spirit of quantum chemistry and uses softer interactions. So far, the present approach has employed $G$ matrices for the description of ground and excited states in ${ }^{4} \mathrm{He}$ [17] and ${ }^{16} \mathrm{O}$ [18], and for nuclei in the vicinity of ${ }^{16} \mathrm{O}[19]$. The most recent calculations are based directly on low-momentum interactions $V_{\text {low } k}$ [32, 33], and the method has been developed to describe weakly bound and unbound helium isotopes within a Gamow-HartreeFock basis [20] and to include 3NF 21].

Within coupled-cluster theory, the ground state of a mass $A$ nucleus is written as

$$
|\psi\rangle=e^{\hat{T}}|\phi\rangle,
$$

where $|\phi\rangle=\prod_{i=1}^{A} \hat{a}_{i}^{\dagger}|0\rangle$ is a single-particle product state and

$$
\hat{T}=\hat{T}_{1}+\hat{T}_{2}+\ldots+\hat{T}_{A}
$$

is a particle-hole $(p-h)$ excitation operator with

$$
\hat{T}_{k}=\frac{1}{(k !)^{2}} \sum_{i_{1}, \ldots, i_{k} ; a_{1}, \ldots, a_{k}} t_{i_{1} \ldots i_{k}}^{a_{1} \ldots a_{k}} \hat{a}_{a_{1}}^{\dagger} \ldots \hat{a}_{a_{k}}^{\dagger} \hat{a}_{i_{k}} \ldots \hat{a}_{i_{1}} .
$$

Here and in the following, $i, j, k, \ldots$ label occupied singleparticle orbitals (as defined by the product state $|\phi\rangle$ ) while $a, b, c, \ldots$ refer to unoccupied orbitals.

We take the reference state $|\phi\rangle$ as our vacuum state and normal-order the Hamiltonian $\hat{H}$ with respect to this state. In practice, we restrict ourselves to the truncation $\hat{T}=\hat{T}_{1}+\hat{T}_{2}$. This is the CCSD approximation, and the coupled-cluster equations are given by

$$
\begin{aligned}
E & =\langle\phi|\bar{H}| \phi\rangle, \\
0 & =\left\langle\phi_{i}^{a}|\bar{H}| \phi\right\rangle, \\
0 & =\left\langle\phi_{i j}^{a b}|\bar{H}| \phi\right\rangle .
\end{aligned}
$$

Here $\left|\phi_{i_{1} \ldots i_{n}}^{a_{1} \ldots a_{n}}\right\rangle=\hat{a}_{a_{n}}^{\dagger} \ldots \hat{a}_{a_{1}}^{\dagger} \hat{a}_{i_{1}} \ldots \hat{a}_{i_{n}}|\phi\rangle$ is a $n p-n h$ excitation of the reference state $|\phi\rangle$, and

$$
\bar{H}=e^{-\hat{T}} \hat{H} e^{\hat{T}}=\left(\hat{H} e^{\hat{T}}\right)_{c}
$$

is the similarity-transformed Hamiltonian (note that $\bar{H}$ is non-Hermitian). The last expression on the righthand side of Eq. (7) indicates that only fully connected diagrams contribute to the construction. The CCSD Eqs. (5) and (6) determine the amplitudes $t_{i}^{a}$ and $t_{i j}^{a b}$ of the $1 p-1 h$ and the $2 p-2 h$ excitation cluster operators, respectively. Once these nonlinear equations are solved, the amplitudes can be inserted into Eq. (4) to determine the ground-state energy.

We remind the reader that an exact solution of the many-body problem would require us to employ the full excitation operator Eq. (2). Such a calculation is as expensive as a full diagonalization of the Hamiltonian, and therefore impossible for medium-mass nuclei. CCSD is very efficient in the sense that it is a highly accurate approximation with the investment of a modest numerical effort that scales as $O\left(n_{o}^{2} n_{u}^{4}\right)$ with the number $n_{o}$ of occupied and the number $n_{u}$ of unoccupied single-particle orbitals, respectively. The inclusion of the $3 p-3 h$ cluster operator $\hat{T}_{3}$ would further increase the accuracy of the method. However, such CCSDT calculations come at the expense $O\left(n_{o}^{3} n_{u}^{5}\right)$ and, at present, are already prohibitively expensive compared to CCSD. For this reason, there is need for more approximate treatments of the full triples equations.

There are various approximations to the full CCSDT equations, and the most popular of these schemes is the $\operatorname{CCSD}(\mathrm{T})$ approach [31]. $\operatorname{CCSD}(\mathrm{T})$ includes diagrams at the CCSDT level that appear up to fifth order in perturbation theory. It is a non-iterative approach since typically converged singles and doubles excitation amplitudes are used in the calculation of the triples energy correction. The $\operatorname{CCSD}(\mathrm{T})$ approximation is relatively inexpensive compared to CCSDT; no storage of triples amplitudes is required and the computational cost is a non-iterative $O\left(n_{o}^{3} n_{u}^{4}\right)$ step. There is also a family of iterative triples correction schemes known as CCSDT$n$ [34]. Their derivation is based on perturbation theory arguments,

$$
\begin{array}{ll}
\text { CCSDT-1 } & 0=\left\langle\phi_{i j k}^{a b c}\left|\left(\hat{F} \hat{T}_{3}+\hat{H} \hat{T}_{2}\right)_{c}\right| \phi\right\rangle, \\
\text { CCSDT-2 } & 0=\left\langle\phi_{i j k}^{a b c}\left|\left(\hat{F} \hat{T}_{3}+\hat{H} \hat{T}_{2}+\hat{H} \hat{T}_{2}^{2} / 2\right)_{c}\right| \phi\right\rangle, \\
\text { CCSDT-3 } & 0=\left\langle\phi_{i j k}^{a b c}\left|\left(\hat{F} \hat{T}_{3}+\hat{H} e^{\hat{T}_{1}+\hat{T}_{2}}\right)_{c}\right| \phi\right\rangle, \\
\text { CCSDT } & 0=\left\langle\phi_{i j k}^{a b c}\left|\left(\hat{H} e^{\hat{T}_{1}+\hat{T}_{2}+\hat{T}_{3}}\right)_{c}\right| \phi\right\rangle .
\end{array}
$$

Here, $\hat{F}$ denotes the Fock operator (the one-body operator that results from the normal ordering of the Hamiltonian). All these approaches require the storage of the full triples amplitudes $t_{i j k}^{a b c}$ and are therefore computationally considerably more expensive than the $\operatorname{CCSD}(\mathrm{T})$ approach. However, for cases where the $\operatorname{CCSD}(\mathrm{T})$ scheme breaks down, one expects the CCSDT- $n$ approaches to perform better. The latter approaches treat the triples corrections self-consistently and also involve the corrections

$$
\begin{array}{r}
\left\langle\phi_{i}^{a}\left|\left(\hat{V} \hat{T}_{3}\right)_{c}\right| \phi\right\rangle, \\
\left\langle\phi_{i j}^{a b}\left|\left(\hat{F} \hat{T}_{3}+\hat{V} \hat{T}_{3}+\hat{V} \hat{T}_{3} \hat{T}_{1}\right)_{c}\right| \phi\right\rangle,
\end{array}
$$

to Eqs. (5) and (6), respectively. These corrections thus modify the values of the amplitudes $t_{i}^{a}$ and $t_{i j}^{a b}$.

\section{B. Low-momentum interactions and model spaces}

Nuclear interactions depend on the resolution scale at which details are probed and resolved. This resolution 
scale dependence is similar to scale and scheme dependences in parton distribution functions. As a result, nuclear interactions are defined by an effective theory for $\mathrm{NN}, 3 \mathrm{~N}$, and many-nucleon interactions and corresponding effective operators,

$$
\hat{V}=V_{\mathrm{NN}}(\Lambda)+V_{3 \mathrm{~N}}(\Lambda)+\ldots,
$$

where the momentum cutoff $\Lambda$ denotes the resolution scale. Conventional nuclear forces are "hard" in the sense that they have large cutoffs that complicate fewand many-body calculations. These difficulties arise from high momenta and associated strong short-range repulsion and short-range tensor forces, which lead to slow convergence with increasing basis size and requires resummations in practice.

Low-momentum interactions $V_{\text {low } k}$ with variable momentum cutoffs show great promise for nuclei [32, 33, 35, 36, 37, 38, 39, 40]. Changing the cutoff leaves lowenergy NN observables unchanged by construction, but shifts contributions between the potential and the sums over intermediate states in loop integrals. These shifts can weaken or largely eliminate sources of nonperturbative behavior such as strong short-range repulsion and the tensor force [36, 41]. An additional advantage is that the corresponding $3 \mathrm{~N}$ interactions become perturbative at lower cutoffs 35] and are thus tractable in coupledcluster theory [21]. The renormalization group (RG) evolution is implemented by coupled RG equations in momentum space 42] or by an equivalent Lee-Suzuki transformation [44, 45].

The evolution to low-momentum interactions $V_{\text {low } k}$ weakens off-diagonal coupling and decouples the lowenergy physics from high-momentum details [46, 47]. As a result, few- and many-body calculations converge more rapidly for lower cutoffs, which is important for extending ab-initio approaches to heavier systems. Finally, the cutoff variation can provide estimates for theoretical uncertainties, which will be left to future work. In this paper, we use a sharp cutoff $\Lambda=1.9 \mathrm{fm}^{-1}$ for the ${ }^{3} \mathrm{H}$ and ${ }^{4} \mathrm{He}$ calculations, and $V_{\text {low } k}$ is derived from the Argonne $v_{18}$ potential [48] in order to benchmark against the Faddeev and Faddeev-Yakubovsky results 35]. For ${ }^{16} \mathrm{O}$ and ${ }^{40} \mathrm{Ca}$, we use a cutoff $\Lambda=2.1 \mathrm{fm}^{-1}$ and compare to the importance-truncated NCSM study [23].

Coupled-cluster theory is employed in a single-particle basis, and we use a model space consisting of spherical harmonic-oscillator states. The basis parameters are the number of orbitals and the oscillator frequency $\hbar \omega$. Our largest model spaces include about $10^{3}$ single-particle orbitals.

\section{RESULTS FOR ${ }^{3} \mathrm{H}$ AND ${ }^{4} \mathrm{HE}$}

In this section, we present our coupled-cluster calculations for the ground-state energies of ${ }^{3} \mathrm{H}$ and ${ }^{4} \mathrm{He}$, and we compare our results to the exact Faddeev and FaddeevYakubovsky energies of Ref. [35]. We first discuss in de-

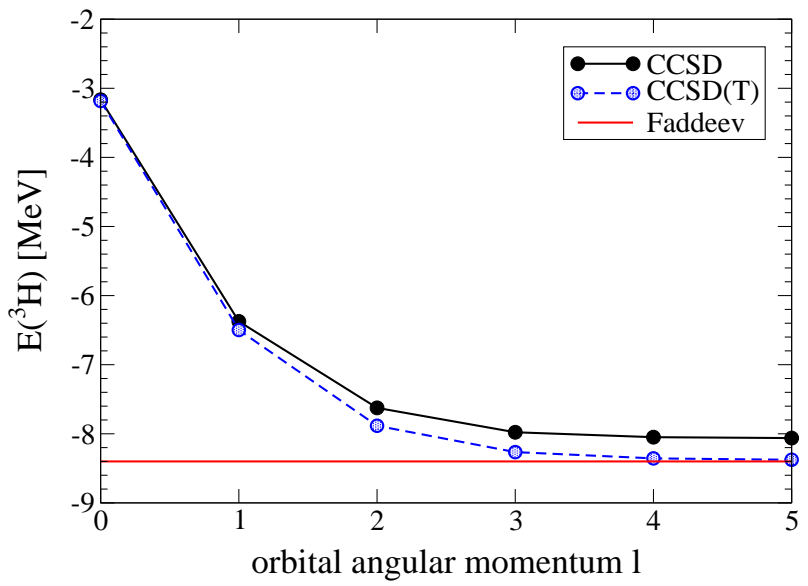

FIG. 1: (Color online) CCSD and CCSD(T) energies for ${ }^{3} \mathrm{H}$ using a model space with fixed maximum $N=2 n+l=12$ and fixed $\hbar \omega=14 \mathrm{MeV}$ as a function of the maximum orbital angular momentum $l$. For comparison, we also show the exact Faddeev result of Ref. [35].

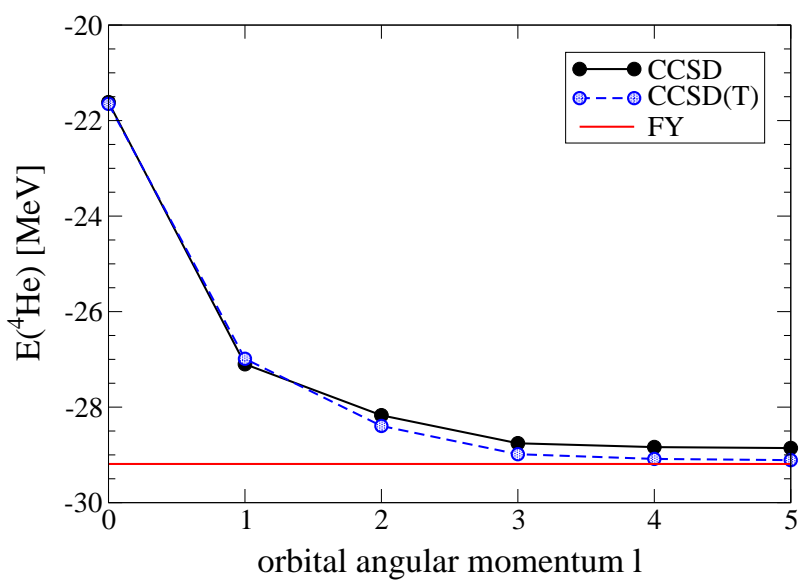

FIG. 2: (Color online) CCSD and CCSD(T) energies for ${ }^{4} \mathrm{He}$ as a function of the maximum orbital angular momentum $l$ and the exact Faddeev-Yakubovsky (FY) result of Ref. 35]. For details, see the caption to Fig. 1

tail the dependence on the size of the model space and the single-particle basis. The coupled-cluster calculations initially used a single-particle basis of oscillator states whose principal and angular momentum quantum numbers $n$ and $l$ obey $2 n+l \leqslant N$, so $N+1$ is the number of major oscillator shells included. Note that in previous calculations [14, 15, 16, 17] $N$ denoted the number of major oscillator shells. However, we observed that the convergence with respect to the angular momentum $l$ is much quicker, since only low partial waves contribute to low-energy properties, while the convergence with respect to the principal quantum number $n$ is slower. This slower convergence is due to the sharp momentum cutoff used for $V_{\text {low } k}$. It is intuitively clear that a harmonic-oscillator representation of an interaction with a sharp cutoff needs a considerable number of radial wave functions to be ac- 


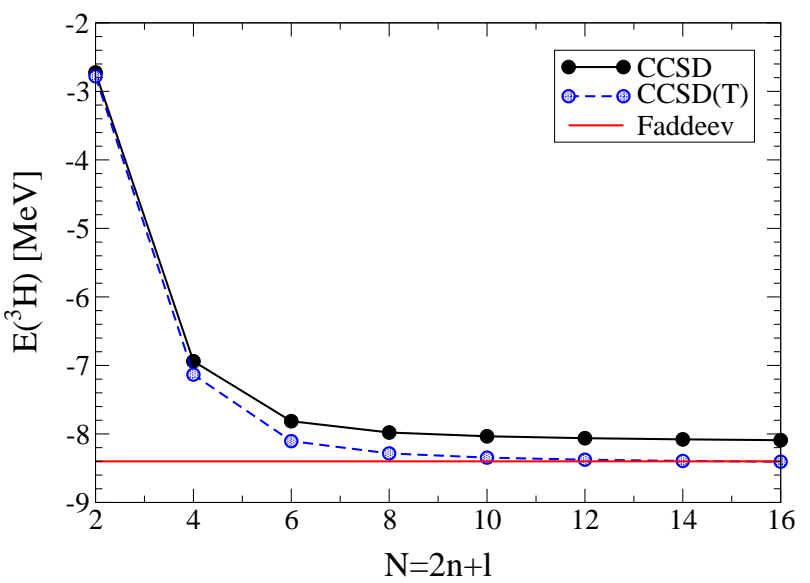

FIG. 3: (Color online) CCSD and $\operatorname{CCSD}(\mathrm{T})$ results for the ground-state energy of ${ }^{3} \mathrm{H}$ as a function of the model-space size $N=2 n+l$, with fixed $l \leqslant 5$ and fixed $\hbar \omega=14 \mathrm{MeV}$. For comparison, we also show the exact Faddeev result of Ref. [35].

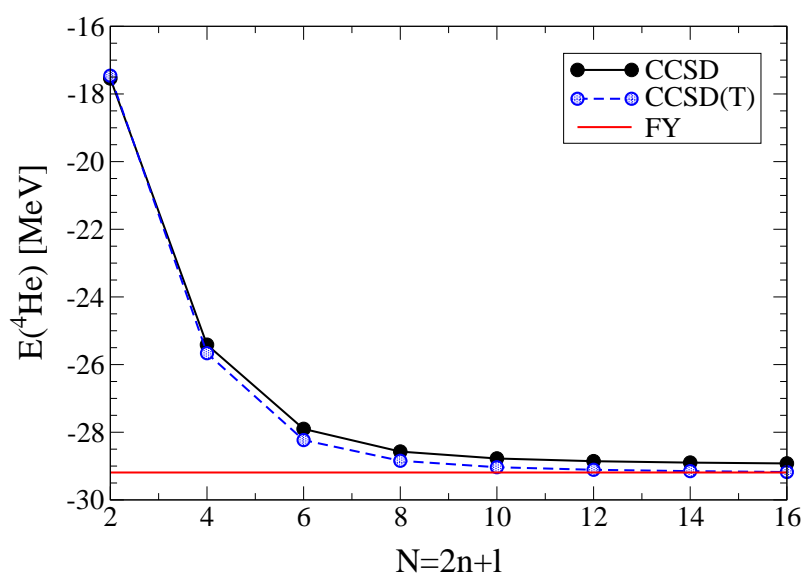

FIG. 4: (Color online) CCSD and CCSD(T) results for the ground-state energy of ${ }^{4} \mathrm{He}$ as a function of the model-space size $N=2 n+l$ and the exact Faddeev-Yakubovsky (FY) result of Ref. [35]. For details, see the caption to Fig. 3 .

curate. The recent work of Refs. 40, 43] confirms this picture and demonstrates that smooth cutoffs improve the convergence in few-body calculations.

Figures 1 and 2 show the convergence of our CCSD and $\operatorname{CCSD}(\mathrm{T})$ energies for ${ }^{3} \mathrm{H}$ and ${ }^{4} \mathrm{He}$ using a model space with fixed $N=2 n+l=12$ and fixed $\hbar \omega=14 \mathrm{MeV}$ as a function of the maximum orbital angular momentum $l$. This implies that for $l=0$ we include oscillator functions with $n \leqslant 6$ nodes; for $l \leqslant 1$ we include oscillator functions with $n \leqslant 6$ for the $s$ states $(l=0)$ and $n \leqslant 5$ for the $p$ states $(l=1)$, and so on. Clearly, the angular momentum quantum number needs not to exceed $l=5$ for the ground-state energies of $s$-shell nuclei. Therefore, we limit our single-particle basis to $l \leqslant 5$ for the following coupled-cluster calculations of ${ }^{3} \mathrm{H}$ and ${ }^{4} \mathrm{He}$.

In Figs. 3 and 4 , we present our CCSD and $\operatorname{CCSD}(\mathrm{T})$

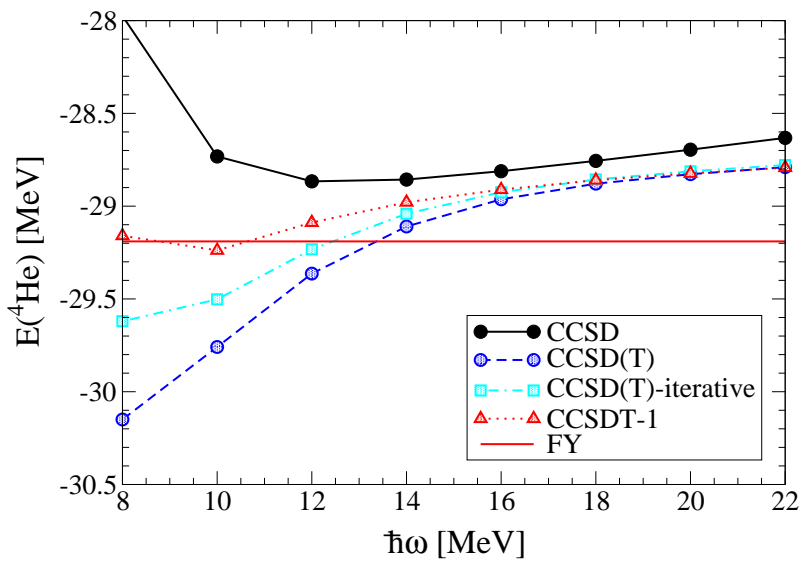

FIG. 5: (Color online) CCSD and various approximate CCSDT energies for ${ }^{4} \mathrm{He}$ as a function of the oscillator frequency $\hbar \omega$, for fixed $N=12$ and $l \leqslant 5$. For comparison, we also show the exact Faddeev-Yakubovsky (FY) result.

results for the ground-state energies of ${ }^{3} \mathrm{H}$ and ${ }^{4} \mathrm{He}$ as a function of the model-space size $N$, with fixed $l \leqslant 5$ and fixed $\hbar \omega=14 \mathrm{MeV}$. Both CCSD and $\operatorname{CCSD}(\mathrm{T})$ energies converge with respect to the model space size for $N \approx$ $12 \ldots 14$. For the largest model space with $N=16$, we obtain for ${ }^{3} \mathrm{H}$,

$$
\begin{aligned}
E_{\mathrm{CCSD}}\left({ }^{3} \mathrm{H}\right) & =-8.09 \mathrm{MeV}, \\
E_{\mathrm{CCSD}(\mathrm{T})}\left({ }^{3} \mathrm{H}\right) & =-8.40 \mathrm{MeV},
\end{aligned}
$$

and for ${ }^{4} \mathrm{He}$,

$$
\begin{aligned}
E_{\mathrm{CCSD}}\left({ }^{4} \mathrm{He}\right) & =-28.92 \mathrm{MeV}, \\
E_{\mathrm{CCSD}(\mathrm{T})}\left({ }^{4} \mathrm{He}\right) & =-29.18 \mathrm{MeV} .
\end{aligned}
$$

The CCSD(T) energies are within $70 \mathrm{keV}$ and $10 \mathrm{keV}$ of the Faddeev and Faddeev-Yakubovsky (FY) results 35] $E\left({ }^{3} \mathrm{H}\right)=-8.470(2) \mathrm{MeV}$ and $E\left({ }^{4} \mathrm{He}\right)=-29.19(5) \mathrm{MeV}$.

Finally, we study the dependence of our results on the oscillator frequency $\hbar \omega$. This is shown in Fig. 5 for fixed $N=12$ and $l \leqslant 5$. While the CCSD results exhibit a very small variation over the shown $\hbar \omega$ range, the variation of the perturbative triples corrections $\operatorname{CCSD}(\mathrm{T})$ is somewhat larger. Moreover, the downward trend of the $\operatorname{CCSD}(\mathrm{T})$ energies with decreasing $\hbar \omega$ indicates that perturbative triples corrections are starting to break down for smaller values of $\hbar \omega$. The non-iterative perturbative triples correction assumes that we work in a basis where the Fock matrix is diagonal. However, our oscillator basis does not diagonalize the Fock matrix, so strict calculations would have to iterate triples corrections until selfconsistency is reached. From Fig. [5 we observe that iterative $\operatorname{CCSD}(\mathrm{T})$ improves on the non-iterative $\operatorname{CCSD}(\mathrm{T})$ results, but also has a downward trend with decreasing $\hbar \omega$. Finally, we present calculations based on the iterative CCSDT-1 approximation to full CCSDT. CCSDT-1 includes all diagrams through fourth order in perturbation theory, but contrary to the perturbative $\operatorname{CCSD}(\mathrm{T})$ 


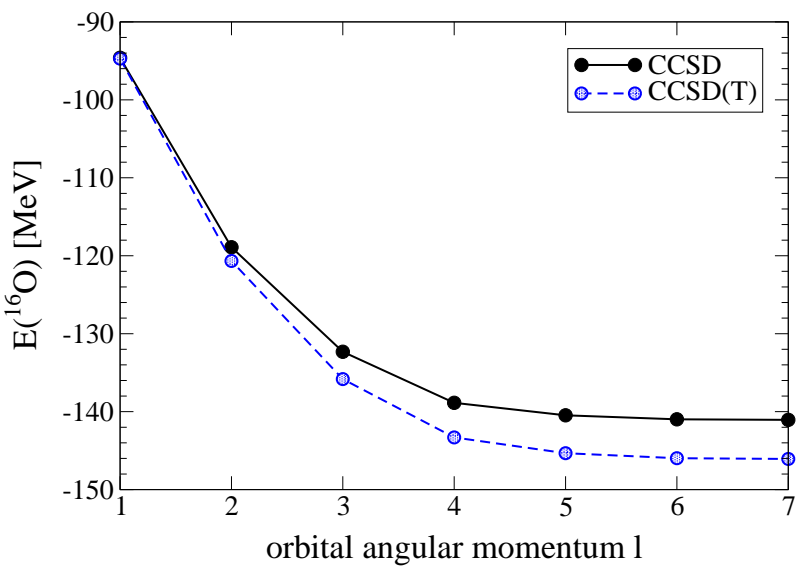

FIG. 6: (Color online) CCSD and CCSD(T) results for the binding energy of ${ }^{16} \mathrm{O}$ using a model space with fixed maximum $N=2 n+l=10$ and fixed $\hbar \omega=20 \mathrm{MeV}$ as a function of the maximum orbital angular momentum $l$.

corrections, the CCSDT-1 approximation is treated selfconsistently and the singles and doubles amplitudes are modified by the triples amplitude according to Eqs. (9) and (10). We clearly find that CCSDT-1 improves on the triples corrections and leads to a very weak dependence on $\hbar \omega$. Note that these CCSDT-1 results are also the first step towards full CCSDT calculations in nuclear physics.

Our results for the light nuclei ${ }^{3} \mathrm{H}$ and ${ }^{4} \mathrm{He}$ demonstrate that coupled-cluster theory meets the benchmarks set by exact methods. It is therefore interesting to use this method to establish benchmark energies for heavier nuclei that other ab-initio approaches can compare to. This is the subject of the next section.

\section{RESULTS FOR ${ }^{16} \mathrm{O}$ AND ${ }^{40} \mathrm{CA}$}

Next, we present our coupled-cluster calculations for ${ }^{16} \mathrm{O}$ and ${ }^{40} \mathrm{Ca}$. In Fig. 6, we show the convergence of the CCSD and $\operatorname{CCSD}(\mathrm{T})$ results for the binding energy of ${ }^{16} \mathrm{O}$ using a model space with fixed $N=2 n+l=10$ and fixed $\hbar \omega=20 \mathrm{MeV}$ as a function of the maximum orbital angular momentum $l$. For ${ }^{16} \mathrm{O}$, we find that $l \leqslant 7$ is sufficient to reach convergence at the $10 \mathrm{keV}$ level. Therefore, we restrict the following coupled-cluster calculations for ${ }^{16} \mathrm{O}$ to $l \leqslant 7$.

Figure 7 shows the dependence of the CCSD binding energies of ${ }^{16} \mathrm{O}$ on the oscillator frequency $\hbar \omega$ for increasing sizes of the model space $N$ with fixed $l \leqslant 7$. The largest calculations for $N=13$ include more than $10^{3}$ single-particle orbitals. We observe that the CCSD energies are converged at the $0.5 \mathrm{MeV}$ level and can be used to extrapolate to infinite model space. This is demonstrated in Fig. 8 where we give the CCSD energies (taken at the $\hbar \omega$ minima) as a function of the model-space size $N$ at fixed $l \leqslant 7$. Using the CCSD minima, we make an exponential fit of the form $E(N)=E_{\infty}+a \exp (-b N)$

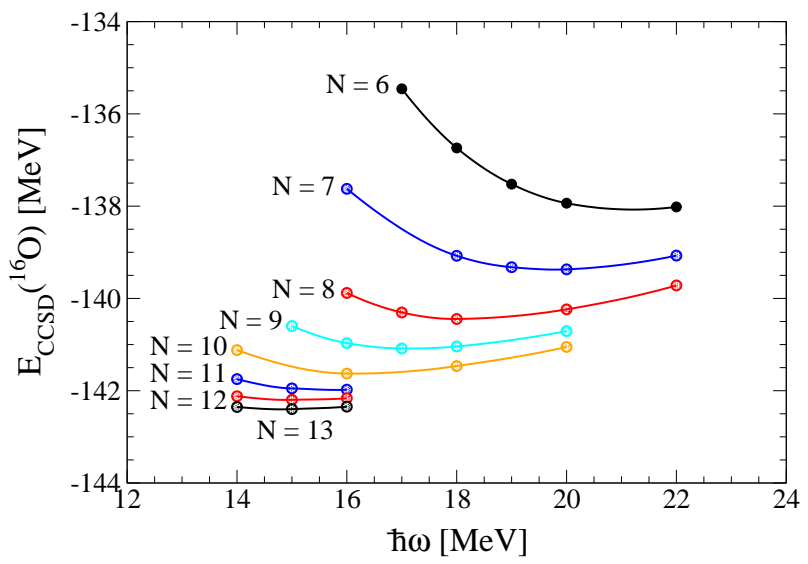

FIG. 7: (Color online) CCSD results for the binding energy of ${ }^{16} \mathrm{O}$ as a function of the oscillator frequency $\hbar \omega$ for increasing sizes of the model space $N=2 n+l$ with fixed $l \leqslant 7$.

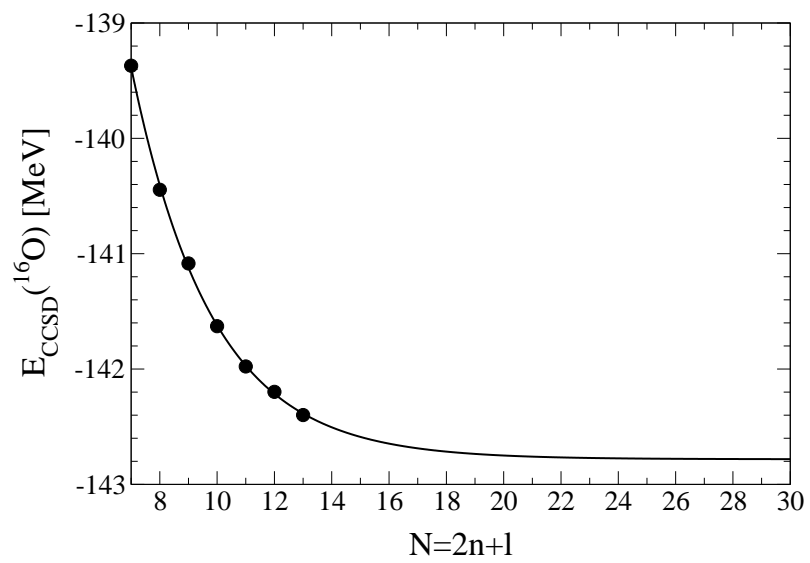

FIG. 8: (Color online) CCSD results (taken at the $\hbar \omega$ minima) for the binding energy of ${ }^{16} \mathrm{O}$ as a function of the model-space size $N=2 n+l$ at fixed $l \leqslant 7$ and exponential fit (solid line).

to the data points. The result is also shown in Fig. 8 and yields the extrapolated infinite model space value $E_{\mathrm{CCSD}, \infty}\left({ }^{16} \mathrm{O}\right)=-142.78 \mathrm{MeV}$. Our largest $N=13$ result is $E_{\mathrm{CCSD}}\left({ }^{16} \mathrm{O}\right)=-142.40 \mathrm{MeV}$. The conservative error estimate due to the finite size of the model space is thus about $0.5 \mathrm{MeV}$.

In Fig. 9, we study triples corrections to the binding energy of ${ }^{16} \mathrm{O}$ via the $\mathrm{CCSD}(\mathrm{T})$ and CCSDT-1 approaches as a function of the oscillator frequency $\hbar \omega$ for increasing sizes of the model space $N(l \leqslant 7)$. We present results up to $N=7$ (eight major oscillator shells), which was the largest model space we could handle for the CCSDT-1 scheme due to storage limitations. The $\operatorname{CCSD}(\mathrm{T})$ and CCSDT-1 energies agree nicely for the range of oscillator frequencies and model spaces considered. The only difference is that, as for ${ }^{4} \mathrm{He}$, the $\operatorname{CCSD}(\mathrm{T})$ approach gives slightly more binding than CCSDT-1 for the largest model spaces. The close agreement between $\operatorname{CCSD}(\mathrm{T})$ and CCSDT-1 gives us confidence that the perturbative triples corrections work well over this regime. 


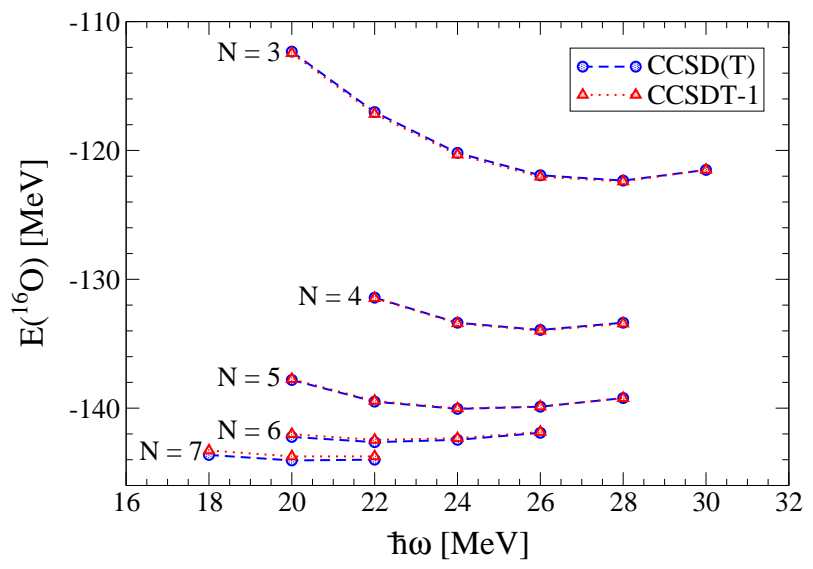

FIG. 9: (Color online) CCSD(T) and CCSDT-1 results for the binding energy of ${ }^{16} \mathrm{O}$ as a function of the oscillator frequency $\hbar \omega$ for increasing sizes of the model space $N=2 n+l(l \leqslant 7)$.

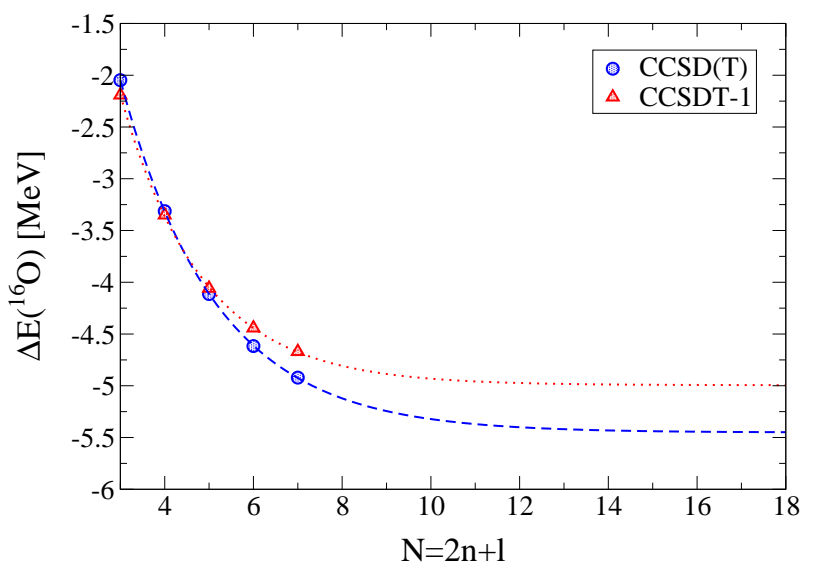

FIG. 10: (Color online) Contributions to the binding energy of ${ }^{16} \mathrm{O}$ from triples corrections $\mathrm{CCSD}(\mathrm{T})$ and CCSDT-1 as a function of the model-space size $N=2 n+l$ at fixed $\hbar \omega=22 \mathrm{MeV}$ and $l \leqslant 7$. The dashed and dotted lines are exponential fits and yield the extrapolated energy corrections.

Let us study the contributions of the triples amplitudes to the binding energy of ${ }^{16} \mathrm{O}$ in more detail. Figure 10 shows the energy differences $\Delta E=E-E_{\mathrm{CCSD}}$ that are due to triples corrections " $(\mathrm{T})$ " and "T- 1 " as a function of the model-space size $N$ at fixed $\hbar \omega=22 \mathrm{MeV}$ and $l \leqslant 7$. The corresponding exponential extrapolations to infinite model spaces yield $-5.45 \mathrm{MeV}$ from the "(T)" correction and $-5.00 \mathrm{MeV}$ from the "T-1" correction. This suggests that the error estimate for the $\operatorname{CCSD}(\mathrm{T})$ and CCSDT1 calculations is about $0.5 \mathrm{MeV}$. This can be viewed as an error estimate due to the truncation of the cluster operator. Combined with the $0.5 \mathrm{MeV}$ uncertainty due to the size of the model space, we thus arrive at an error estimate of about $1 \mathrm{MeV}$ for ${ }^{16} \mathrm{O}$.

We note that ${ }^{16} \mathrm{O}$ is overbound by about $20 \mathrm{MeV}$ when compared to the experimental binding energy. A similar result has been found by Fujii et al. [49]. However, a comparison of results based only on NN interactions to

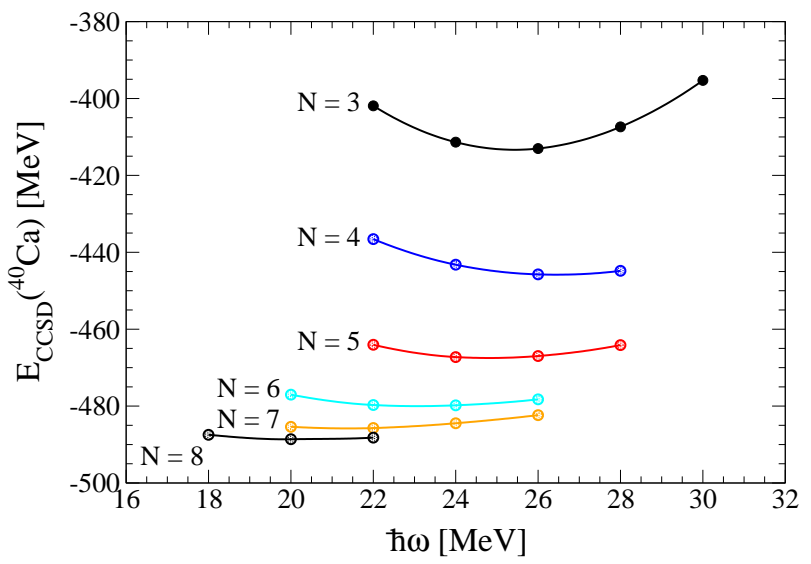

FIG. 11: (Color online) CCSD results for the binding energy of ${ }^{40} \mathrm{Ca}$ as a function of the oscillator frequency $\hbar \omega$ for increasing sizes of the model space $N=2 n+l$. (Note that there is no restriction on $l$ for these model spaces.)

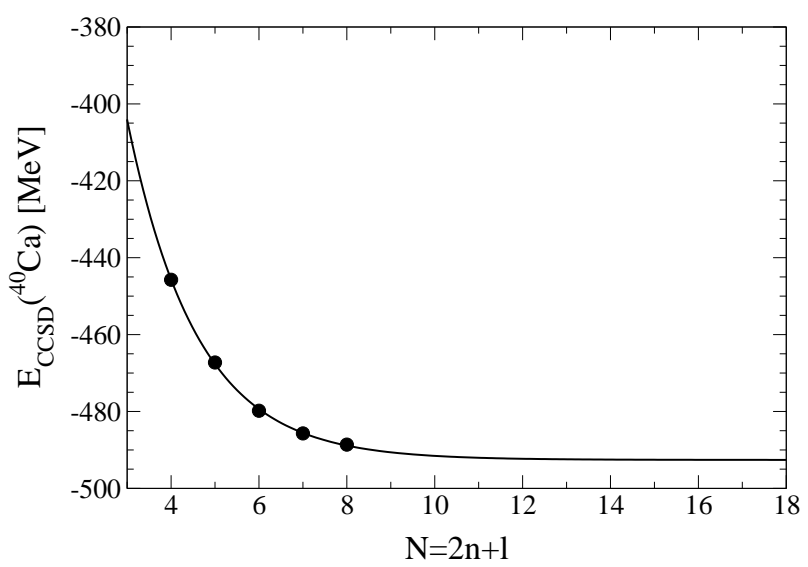

FIG. 12: (Color online) CCSD results (taken at the $\hbar \omega$ minima) for the binding energy of ${ }^{40} \mathrm{Ca}$ as a function of the modelspace size $N=2 n+l$ (without restriction in $l$ ) and exponential fit (solid line).

experiment is meaningless, since $3 \mathrm{NF}$ are crucial to describe few- and many-body observables (see for instance the discussion in Refs. 21, 35, 36]). In nuclear matter, the corresponding $3 \mathrm{NF}$ contribution is repulsive and the expectation values remain consistent with chiral effective field theory power-counting estimates [36].

Finally, we turn to the more challenging case of ${ }^{40} \mathrm{Ca}$. Figure 11] shows the CCSD binding energy of ${ }^{40} \mathrm{Ca}$ as a function of the oscillator frequency $\hbar \omega$ for model spaces up to $N=8$ (nine major oscillator shells). (Note that there is no restriction on $l$ for these model spaces.) This represents the largest coupled-cluster calculation to date in nuclear physics. In these largest calculations, we have 40 active particles in 660 single-particle orbitals. The effective shell-model dimension in this space would be of the order of $10^{63}$. From Fig. [11, we find that the CCSD energies of ${ }^{40} \mathrm{Ca}$ are converging reasonably well. We again expect that low-momentum interactions with 


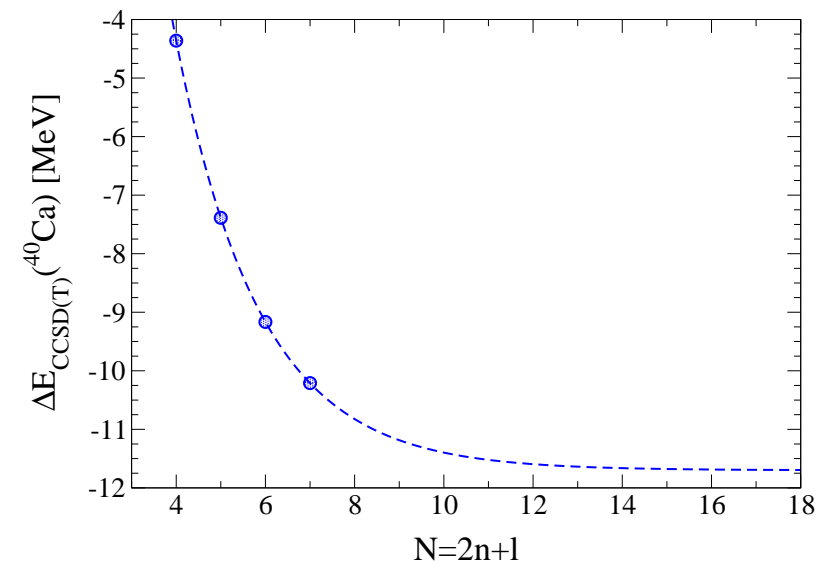

FIG. 13: (Color online) Contributions to the binding energy of ${ }^{40} \mathrm{Ca}$ from triples corrections $\operatorname{CCSD}(\mathrm{T})$ as a function of the model-space size $N=2 n+l$ at fixed $\hbar \omega=22 \mathrm{MeV}$ (without restriction in $l$ ) and exponential fit (dashed line).

smooth cutoffs will lead to even improved convergence. In Fig. 12, we present the CCSD energies for ${ }^{40} \mathrm{Ca}$ (taken at the $\hbar \omega$ minima) as a function of the model-space size $N$. The exponential extrapolation to infinite model space yields $E_{\mathrm{CCSD}, \infty}\left({ }^{40} \mathrm{Ca}\right)=-492.6 \mathrm{MeV}$, and we find that the CCSD energy for $N=8$ is about $4 \mathrm{MeV}$ from the fully converged CCSD result.

We then perform $\operatorname{CCSD}(\mathrm{T})$ calculations and show in Fig. 13 the triples energy corrections as a function of the model-space size $N$ at fixed $\hbar \omega=22 \mathrm{MeV}$. Due to memory limitations, we were not able to perform CCSDT1 calculations in model spaces reaching up to $N=7$. The exponential extrapolation to infinite model space yields $-11.70 \mathrm{MeV}$, while the largest $N=7$ result is $-10.21 \mathrm{MeV}$. The convergence we find with respect to the size of the model space is similar to the recent results by Fujii et al. [50]. Recall that the different triples corrections for ${ }^{16} \mathrm{O}$ differed by about $10 \%$ from each other. Thus, we estimate that the error due to the truncation of the cluster operator is about $1 \mathrm{MeV}$ for ${ }^{40} \mathrm{Ca}$. The total error estimate for ${ }^{40} \mathrm{Ca}$ is thus about $5 \mathrm{MeV}$, and is dominated by the uncertainty due to the finite size of the model space.

We summarize our coupled-cluster results for the binding energies of ${ }^{4} \mathrm{He},{ }^{16} \mathrm{O}$, and ${ }^{40} \mathrm{Ca}$ in Table I] which gives the extrapolated correlation energies $\Delta E_{\mathrm{CCSD}}$ and $\Delta E_{\mathrm{CCSD}(\mathrm{T})}$. We find that for ${ }^{4} \mathrm{He},{ }^{16} \mathrm{O}$, and ${ }^{40} \mathrm{Ca}$, the triples corrections are a factor of $\approx 0.015,0.066$, and 0.081 smaller than the CCSD correlation energies. From this, we again estimate the missing correlation energy from quadruples, pentuplets, and so on, to be of the order of $1 \mathrm{MeV}$ for ${ }^{40} \mathrm{Ca}$. We note that ${ }^{16} \mathrm{O}$ is overbound by about $20 \mathrm{MeV}$ and ${ }^{40} \mathrm{Ca}$ by about $150 \mathrm{MeV}$ when compared to the experimental binding energies. This is not surprising and points to the importance of $3 \mathrm{NF}$ for nuclear structure calculations [21, 35, 36].

We can compare the coupled-cluster energies to the recent importance-truncated NCSM results of Roth and

\begin{tabular}{l|r|r|r}
\hline & \multicolumn{1}{|c|}{${ }^{4} \mathrm{He}$} & \multicolumn{1}{c}{${ }^{16} \mathrm{O}$} & ${ }^{40} \mathrm{Ca}$ \\
\hline \hline$E_{0}$ & -11.8 & -60.2 & -347.5 \\
$\Delta E_{\mathrm{CCSD}}$ & -17.1 & -82.6 & -143.7 \\
$\Delta E_{\mathrm{CCSD}(\mathrm{T})}$ & -0.3 & -5.4 & -11.7 \\
\hline$E_{\mathrm{CCSD}(\mathrm{T})}$ & -29.2 & -148.2 & -502.9 \\
\hline \hline exact (FY) & $-29.19(5)$ & & \\
\hline
\end{tabular}

TABLE I: Reference vacuum energies, $E_{0}$, CCSD and $\mathrm{CCSD}(\mathrm{T})$ correlation energies, $\Delta E_{\mathrm{CCSD}}$ and $\Delta E_{\mathrm{CCSD}(\mathrm{T})}$, and binding energies $E_{\mathrm{CCSD}(\mathrm{T})}$ for ${ }^{4} \mathrm{He},{ }^{16} \mathrm{O}$ and ${ }^{40} \mathrm{Ca}$. The vacuum energies, $E_{0}$, are for $\hbar \omega=14 \mathrm{MeV}$ in the case of ${ }^{4} \mathrm{He}$ and $\hbar \omega=22 \mathrm{MeV}$ for ${ }^{16} \mathrm{O}$ and ${ }^{40} \mathrm{Ca}$. The CCSD and $\operatorname{CCSD}(\mathrm{T})$ energies are the extrapolated infinite model space results. The exact Faddeev-Yakubovsky result is from Ref. 35]

Navrátil [23] which are based on the same $V_{\text {low } k}$ interaction. The importance-truncated NCSM combines a particle-hole truncation scheme $\left(4 p-4 h\right.$ for ${ }^{16} \mathrm{O}$ and $3 p-3 h$ for ${ }^{40} \mathrm{Ca}$ in Ref. [23]) with importance sampling of manybody states based on perturbation theory. The particlehole truncation scheme leads to unlinked diagrams and hence is not size-extensive [22]. Using an exponential extrapolation, Roth and Navrátil 23] find binding energies $E=-137.75 \mathrm{MeV}$ for ${ }^{16} \mathrm{O}$ and $E=-461.83 \mathrm{MeV}$ for ${ }^{40} \mathrm{Ca}$ at the minimum in $\hbar \omega$. Our coupled-cluster results idicate that the converged energies are approximately $10 \mathrm{MeV}$ and $40 \mathrm{MeV}$ lower for ${ }^{16} \mathrm{O}$ and ${ }^{40} \mathrm{Ca}$, respectively. Note also that CCSD scales computationally more favorably than a full $4 p$ - $4 h$ calculation, while it already includes a considerable part of linked $4 p-4 h$ excitations [22].

\section{SUMMARY}

In summary, we have performed ab-initio coupledcluster calculations for ${ }^{3} \mathrm{H},{ }^{4} \mathrm{He},{ }^{16} \mathrm{O}$, and ${ }^{40} \mathrm{Ca}$ based on low-momentum interactions $V_{\text {low } k}$. At the $\operatorname{CCSD}(\mathrm{T})$ level, the ground-state energies for ${ }^{3} \mathrm{H}$ and ${ }^{4} \mathrm{He}$ are practically converged with respect to the size of the modelspace and exhibit a very weak dependence on the oscillator frequency. The resulting energies are within $70 \mathrm{keV}$ and $10 \mathrm{keV}$ of the corresponding Faddeev and FaddeevYakubovsky benchmarks. For ${ }^{16} \mathrm{O}$ and ${ }^{40} \mathrm{Ca}$, we estimate that the $\operatorname{CCSD}(\mathrm{T})$ binding energies are converged within $1 \mathrm{MeV}$ and $5 \mathrm{MeV}$, respectively. Future calculations will include convergence studies for low-momentum interactions with smooth cutoffs [43, 46] and advancing the $3 \mathrm{NF}$ frontier to medium-mass nuclei based on the findings of Ref. [21]. Our results confirm that coupled-cluster theory is a powerful $a b$-initio method that meets and sets benchmarks. 


\section{Acknowledgments}

We thank S.K. Bogner, R.J. Furnstahl and A. Nogga for useful discussions. This work was supported by the U.S. Department of Energy under Contract Nos. DEAC05-00OR22725 with UT-Battelle, LLC (Oak Ridge National Laboratory), and DE-FC02-07ER41457 (University of Washington), and under Grant No. DE-FG02-
96ER40963 (University of Tennessee), and by the Natural Sciences and Engineering Research Council of Canada (NSERC). TRIUMF receives federal funding via a contribution agreement through the National Research Council of Canada. Computational resources were provided by the National Center for Computational Sciences at Oak Ridge and the National Energy Research Scientific Computing Facility.
[1] H. Kamada, A. Nogga, W. Glöckle, E. Hiyama, M. Kamimura, K. Varga, Y. Suzuki, M. Viviani, A. Kievsky, S. Rosati, J. Carlson, S.C. Pieper, R.B. Wiringa, P. Navrátil, B.R. Barrett, N. Barnea, W. Leidemann, and G. Orlandini, Phys. Rev. C 64, 044001 (2001), nucl-th/0104057

[2] A. Nogga, H. Kamada, W. Glöckle, Phys. Rev. Lett. 85, 944 (2000), nucl-th/0004023.

[3] K. Varga and Y. Suzuki, Phys. Rev. C 52, 2885 (1995), nucl-th/9508023

[4] E. Hiyama, Y. Kino and M. Kamimura, Prog. Part. Nucl. Phys. 51, 223 (2003), nucl-th/0402007.

[5] M. Viviani, A. Kievsky and S. Rosati, Phys. Rev. C 71, 024006 (2005), nucl-th/0408019.

[6] M. Viviani, L.E. Marcucci, S. Rosati, A. Kievsky and L. Girlanda, Few Body Syst. 39, 159 (2006), nucl-th/0512077.

[7] S.C. Pieper and R.B. Wiringa, Ann. Rev. Nucl. Part. Sci. 51, 53 (2001), nucl-th/0103005

[8] P. Navrátil, J.P. Vary, and B.R. Barrett, Phys. Rev. C 62, 054311 (2000).

[9] P. Navrátil, V.G. Gueorguiev, J.P. Vary, W.E. Ormand, and A. Nogga, nucl-th/0701038.

[10] N. Barnea, W. Leidemann and G. Orlandini, Phys. Rev. C 61, 054001 (2000), nucl-th/9910062

[11] D. Gazit, S. Bacca, N. Barnea, W. Leidemann and G. Orlandini, Phys. Rev. Lett. 96, 112301 (2006), nucl-th/0512038

[12] F. Coester, Nucl. Phys. 7, 421 (1958).

[13] F. Coester and H. Kümmel, Nucl. Phys. 17, 477 (1960).

[14] J.H. Heisenberg and B. Mihaila, Phys. Rev. C 59, 1440 (1999), nucl-th/9802029.

[15] B. Mihaila and J.H. Heisenberg, Phys. Rev. C 61, 054309 (2000), nucl-th/9912023, Phys. Rev. Lett. 84, 1403 (2000), nucl-th/9910007.

[16] D.J. Dean and M. Hjorth-Jensen, Phys. Rev. C 69, 054320 (2004), nucl-th/0308088.

[17] K. Kowalski, D.J. Dean, M. Hjorth-Jensen, T. Papenbrock, and P. Piecuch, Phys. Rev. Lett. 92, 132501 (2004), nucl-th/0310082.

[18] M. Włoch, D.J. Dean, J.R. Gour, M. Hjorth-Jensen, K. Kowalski, T. Papenbrock, and P. Piecuch, Phys. Rev. Lett. 94, 212501 (2005), nucl-th/0501067.

[19] J.R. Gour, P. Piecuch, M. Hjorth-Jensen, M. Włoch, and D.J. Dean, Phys. Rev. C 74, 024310 (2006), nucl-th/0507049

[20] G. Hagen, D.J. Dean, M. Hjorth-Jensen, and T. Papenbrock, nucl-th/0610072.

[21] G. Hagen, T. Papenbrock, D.J. Dean, A. Schwenk, A. Nogga, P. Piecuch and M. Włloch, arXiv:0704.2854.

[22] R.J. Bartlett and M. Musiał, Rev. Mod. Phys. 79, 291
(2007).

[23] R. Roth and P. Navrátil, arXiv:0705.4069.

[24] J. Paldus and X. Li, Adv. Chem. Phys. 110, 1 (1999).

[25] T.D. Crawford and H.F. Schaefer III, Rev. Comput. Chem. 14, 33 (2000).

[26] P. Piecuch, K. Kowalski, I.S.O. Pimienta, P.-D. Fan, M. Lodriguito, M.J. McGuire, S.A. Kucharski, T. Kus, and M. Musial, Theor. Chem. Acc. 112, 349 (2004).

[27] H. Kümmel, K.H. Lührmann, and J.G. Zabolitzky, Phys. Rep. 36, 1 (1978).

[28] R. F. Bishop, M. F. Flynn, M. C. Boscá, E. Buendá, and R. Guardiola, Phys. Rev. C 42, 1341 (1990).

[29] J. Čížek, J. Chem. Phys. 45, 4256 (1966).

[30] J. Č́žzek, Adv. Chem. Phys. 14, 35 (1969).

[31] M.J.O. Deegan and P.J. Knowles, Chem. Phys. Lett. 227, 321 (1994).

[32] S.K. Bogner, T.T.S. Kuo, A. Schwenk, D.R. Entem, and R. Machleidt, Phys. Lett. B 576, 265 (2003), nucl-th/0108041

[33] S.K. Bogner, T.T.S. Kuo, and A. Schwenk, Phys. Rept. 386, 1 (2003), nucl-th/0305035

[34] Y.S. Lee, S.A. Kucharski, R.J. Bartlett, J. Chem. Phys. 81, 5906 (1984); ibid 82, 761 (E) (1982). J. Noga, R.J. Bartlett, M. Urban, Chem. Phys. Lett. 134, 126 (1987).

[35] A. Nogga, S.K. Bogner, and A. Schwenk, Phys. Rev. C 70, 061002(R) (2004), nucl-th/0405016.

[36] S.K. Bogner, A. Schwenk, R.J. Furnstahl, and A. Nogga, Nucl. Phys. A 763, 59 (2005), nucl-th/0504043

[37] S.K. Bogner, T.T.S. Kuo, L. Coraggio, A. Covello and N. Itaco, Phys. Rev. C 65, 051301 (2002).

[38] A. Schwenk and A.P. Zuker, Phys. Rev. C 74, 061302 (2006), nucl-th/0501038.

[39] S.K. Bogner and R.J. Furnstahl, Phys. Lett. B 632, 501 (2006), nucl-th/0508022

[40] S.K. Bogner and R.J. Furnstahl, Phys. Lett. B 639, 237 (2006), nucl-th/0602017.

[41] S.K. Bogner, R.J. Furnstahl, S. Ramanan and A. Schwenk, Nucl. Phys. A 773, 203 (2006), nucl-th/0602060

[42] S.K. Bogner, A. Schwenk, T.T.S. Kuo and G.E. Brown, nucl-th/0111042.

[43] S.K. Bogner, R.J. Furnstahl, S. Ramanan, and A. Schwenk, Nucl. Phys. A 784, 79 (2007), nucl-th/0609003.

[44] S.Y. Lee and K. Suzuki, Phys. Lett. B91, 173 (1980).

[45] K. Suzuki and S.Y. Lee, Prog. Theor. Phys. 64, 2091 (1980).

[46] S.K. Bogner, R.J. Furnstahl and R.J. Perry, Phys. Rev. C 75, 061001 (2007), nucl-th/0611045.

[47] S.K. Bogner, R.J. Furnstahl, R.J. Perry and A. Schwenk, Phys. Lett. B 649, 488 (2007), nucl-th/0701013

[48] R.B. Wiringa, V.G.J. Stoks, and R. Schiavilla, Phys. 
Rev. C 51, 38 (1995).

[49] S. Fujii, H. Kamada, R. Okamoto and K. Suzuki, nucl-th/0406082
[50] S. Fujii, R. Okamoto, K. Suzuki, J. Phys. Conf. Ser. 20, 83 (2005), nucl-th/0505076. 